\title{
Evaluation of Free Form Deformation and Demons Registration with Discontinuities
}

\author{
Emir SKEJIĆ, Damir DEMIROVIĆ, Dušan GLEICH, Amira ŠERIFOVIĆ-TRBALIĆ
}

\begin{abstract}
Medical image registration plays an important part in most today's clinical procedures. Registration goal is to find transformation which warps one image into the space of another. Registration of moving organs in human body has a significant part in therapy planning. This task is harder in cases when one organ (tissue) slides along another, i.e. in a case of discontinuities in the motion field. Discontinuities introduce unwanted transformations which often lead to poor or unsatisfied registration results. In this paper we evaluate one form of discontinuities for two well-known and used registration algorithms namely Free Form Deformation and Demons.
\end{abstract}

Keywords: demons algorithm; discontinuities; free form deformation; medical image registration

\section{INTRODUCTION}

Image registration is the process of overlaying two or more images of the same scene taken at different times, from different viewpoints, and/or by different sensors. The goal of registration is to find spatial alignment of images [1]. Establishing the correspondences of spatial information in medical images and equivalent structures in the body is fundamental to medical image interpretation and analysis. The images might be acquired with different sensors or the same sensor at different times. Radiologist with subjective judgment of relative size, shape, and spatial relationships of visible structures and physiology inferred from intensity distributions, uses this information for developing a diagnosis, planning a therapy, and monitoring the disease progression or response. During the previous twenty years a number of image registration algorithms were proposed.

In Thirion [2] was proposed a method for non/rigid image registration. Rueckert [3] proposed the use of Free Form Deformations for non/rigid image registration. During the time, several improvements regarding the precision and registration speed were developed. In Muyan-Ozcelik [4], GPU implementation of Demons algorithm was developed. Image registration is a high time consuming operation. In this paper, Demons registration for 3D CT lung images was developed on a Graphics Processing Units (GPU), wherein speed improvements of 55 times were reported. Better description of Demons algorithm can be found in Penec [5]. In $\mathrm{Gu}$ [6], five variants of demons algorithm were developed on a GPU. In Zhao [7] a framework for capturing very large deformations was developed using demons algorithm.

In Shi [8] was proposed a sparse Free Form Deformation for which fine local details such as motion discontinuities could be captured. In Yang [9], demons algorithm with locally adaptive regularization was proposed.

In this paper, two widely used image registration algorithms are analysed for the case of motion discontinuities, and those are Free Form Deformations and Demons algorithm.

\section{METHODS}

Image registration process can be described as in Fig. 1. In general, image registration needs two images, here called moving and fixed. As an output of registration algorithm we have registered image. During registration, an algorithm iteratively transforms moving image to match fixed image. Final transformed image should be "similar" to fixed image (Fig. 2). Similarity is calculated using the criterion which usually compares corresponding pixels from input images, and also terminates the registration process. Image registration has applications in many fields; one addressed in this paper deals with medical imaging, specifically radiological imaging. In this section we will give a review for the two most used algorithms for non-rigid image registration.

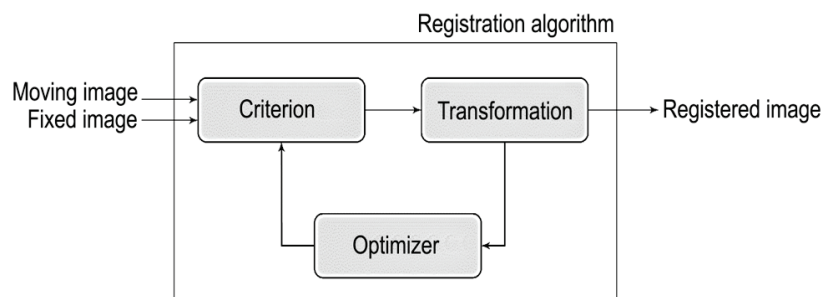

Figure 1 Three components of the image registration algorithm

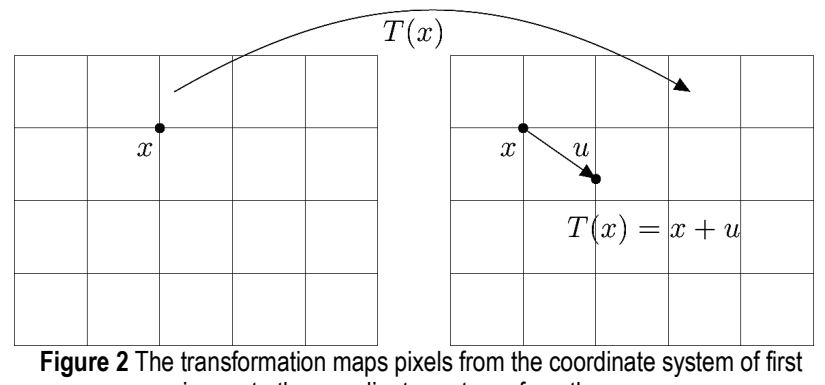
image to the coordinate system of another one.

\subsection{Free Form Deformations}

Free Form Deformations algorithm uses Thin Plate Splines $[10,11,12]$. A thin-plate spline (TPS) belongs to the family of splines based on radial basis functions. In medical image registration, thin-plate splines are widely used, for example, as an approximation solution where the degree of approximation depends on the confidence of landmark localization. They have been formed by Duchon and Meinguet for the surface interpolation of scattered data [10,11]. Radial basis function (RBF) spline can be defined as a linear combination of $n$ radial basis functions $\theta(s)$. 


$$
t(x, y, z)=a_{1}+a_{2} x+a_{3} y+a_{4} z+\sum_{j=1}^{n} b_{j} \theta\left(\left|\phi_{j}-(x, y, z)\right|\right)(1)
$$

The transformation can be defined as three separate thin plate spline functions $T=\left(t_{1}, t_{2}, t_{3}\right)^{\mathrm{T}}$ (Eq. (1)), which yield mapping between images where the coefficients correspond to a fine part of mapping of the spline-based transformation.

The goal of free-form deformations is to provide the means for modelling arbitrary deformations applied to objects. The general idea is to deform an image by manipulating a regular grid of control points that are distributed across the image. The control points can be moved, and the position of the individual pixels between control points is computed from positions of surrounding points. Compared to other type of splines (thin-plate spline, elastic-body spline), B-splines are locally controlled. This fact leads to computational efficiency for large number of points. In [3] was presented the framework for FFD registering breast images, which consists of combined local and global transformation (Eq. (2)).

Combined transform is defined as the following

$$
T(x, y, z)=T_{\text {global }}(x, y, z)+T_{\text {local }}(x, y, z)
$$

FFD is deformed an object by manipulating an underlying mesh of control points. On the image volume, we define the mesh of control points with uniform spacing. FFD can be defined as a 3D tensor product of 1D cubic B-splines:

$$
T_{l o c a l}(x, y, z)=\sum_{l=0}^{3} \sum_{m=0}^{3} \sum_{n=0}^{3} B_{l}(u) B_{m}(v) B_{n}(\omega) \phi_{(i+l, j+m, k+n)},
$$

where:

$$
\begin{aligned}
& i=\left\lfloor x / n_{x}\right\rfloor-1, j=\left\lfloor y / n_{y}\right\rfloor-1, \\
& k=\left\lfloor z / n_{z}\right\rfloor-1, u=x / n_{x}-\left\lfloor x / n_{x}\right\rfloor, \\
& v=y / n_{y}-\left\lfloor y / n_{y}\right\rfloor, \omega=z / n_{z}-\left\lfloor z / n_{z}\right\rfloor .
\end{aligned}
$$

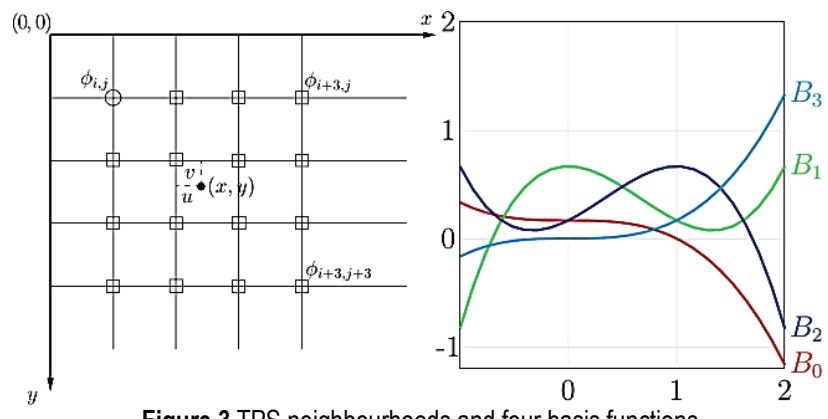

Figure 3 TPS neighbourhoods and four basis functions

Here $B_{l}$ represents $l^{\text {th }}$ basis function of the B-spline (Fig. 3).

$$
\begin{aligned}
& B_{0}(u)=(1-u)^{3} / 6 \\
& B_{1}(u)=\left(3 u^{3}-6 u^{2}+4\right) / 6 \\
& B_{2}(u)=\left(-3 u^{3}+3 u^{2}+3 u+1\right) / 6 \\
& B_{3}(u)=u^{3} / 6
\end{aligned}
$$

An advantage of FFD is a number of degrees of freedom, which has greater significance in non-rigid registration cases. Spacing of control points in the algorithm defines the number of degrees of freedom and computational complexity. Multi-resolution approach as described in Forsey [13] can be applied. Here we have hierarchy of control point meshes at different resolutions, whereas the resolution increases at each resolution level. This way, the sum of local transformations gives the local transformation. To obtain smooth transformations, this spline-based FFD is constrained with the regularizer, as defined in Wahba [14]. The part of cost function which models TPS is defined with the following

$$
\begin{aligned}
& C_{\text {smooth }}=\frac{1}{V} \int_{0}^{X} \int_{0}^{Y} \int_{0}^{Z}\left[\left(\frac{\partial^{2} T}{\partial x^{2}}\right)^{2}+\left(\frac{\partial^{2} T}{\partial y^{2}}\right)^{2}+\left(\frac{\partial^{2} T}{\partial z^{2}}\right)^{2}+\right. \\
& \left.+2\left(\frac{\partial^{2} T}{\partial x y}\right)^{2}+\left(\frac{\partial^{2} T}{\partial x z}\right)^{2}+\left(\frac{\partial^{2} T}{\partial y z}\right)^{2}\right] d x d y d z
\end{aligned}
$$

Therefore, the cost function is defined as the following

$$
C(\Theta, \Phi)=-C_{\text {similarity }}\left(I\left(t_{0}\right), T(I(t))\right)+\lambda C_{\text {smooth }}(T)
$$

The smoothness part in Eq. (4) is the binding energy of thin-plate of metal. Minimizing the previous cost function, we find an "optimal" transformation. In the previous equation, the term $C_{\text {similarity }}$ is the image similarity defined with mutual information, and the second term $\lambda C_{\text {smooth }}$ corresponds to the cost associated with the smoothness of transformation. Finding optimum of Eq. (5) can be done with some of the well-known optimization methods, for example Gradient descent method.

\section{DEMONS ALGORITHM}

Thirion [3] has proposed the images to non-rigidly register by means of the diffusion process. In that paper he introduced the image entities (called demons) that push according to local characteristics of images in the similar way Maxwell did for solving the Gibbs paradox in thermodynamics. Basically, the task for demons is to sort the particles. This process moves particles outwards and inwards, which depends on the relation of scene and model. A good description of the Demons algorithm is given in Pennec [5]. We can illustrate the idea for twodimensional case with two images $S$ and $M$ (Fig. 4). A demon $d$ is located at spatial position $d$ and $R(d) \neq 0$.

According to the gradient $R(d)$ and the image difference $S(d)-M(d)$, the demons produce the pushing force $p$. Template is pushed according to $S(d)$, if $S(d)<M(d)$ and $-S(d)$, if $S(d)>M(d)$. Using gradient information 
for expressing the demons forces may not be efficient when gradient values are close to 0 . For the estimation of demons forces, optical flow equation is used. For a given point $P$, let $s$ be the intensity in static image $S$ and $m$ the intensity of the moving image $M$. Then the estimated displacement $u$ required for the point is

$$
\vec{u}=\frac{(m-s) \vec{\nabla} s}{|\vec{\nabla} s|^{2}+(m-s)^{2}}
$$

Here $\vec{u}=\left(u_{x}, u_{y}, u_{z}\right)$ are components of the displacement, $\nabla S$ is a gradient of the static image. Here $\nabla S$ represents 'internal' force originating from the static image. The term $(m-s)^{2}$ is differential force of the interaction between the static and moving image which is an 'external' force. Thirion has proposed iterative calculation of Eq. (6). The displacement is regularized in each iteration by Gaussian filter with a variance of $\sigma^{2}$. Here the regularization plays an important role in preserving the geometric continuity and suppressing the noise.

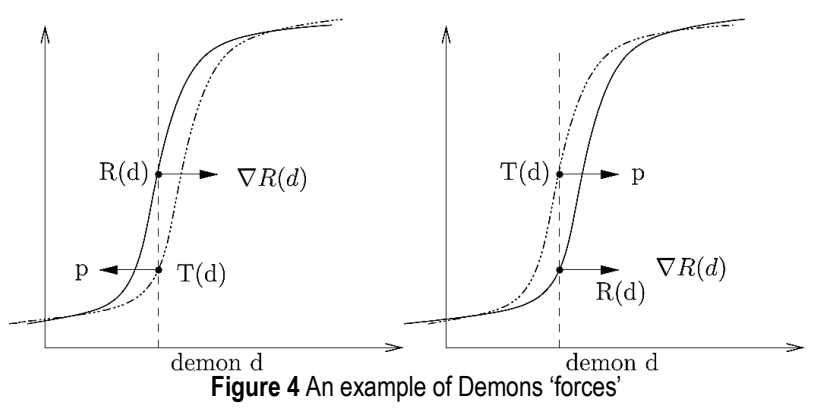

From Eq. (6) it can be seen that information is driven only from static image by using its gradient. In [15] authors propose to expand the original method for calculating demons with another 'active' part or force $\vec{f}_{m}$, which is based on the gradient information from the moving image

$\vec{f}_{m}=\frac{(m-s) \vec{\nabla} m}{|\vec{\nabla} m|^{2}+(\mathrm{s}-m)^{2}}$

By combining Eq. (6) and (7) we have the total force expressed as

$$
\vec{f}=(\mathrm{m}-s) \times\left(\frac{\vec{\nabla} s}{|\vec{\nabla} s|^{2}+(\mathrm{s}-m)^{2}}+\frac{\vec{\nabla} m}{|\vec{\nabla} m|^{2}+(\mathrm{s}-m)^{2}}\right)
$$

Here the influences of 'passive' and 'active' forces are mixed, whereas 'active' force is used to emphasize the contribution of the gradient information from moving image. Normalization factor $\alpha$ enables to adjust the force strength in each iteration, and smaller $\alpha$ can be used for relatively large deformations and then reduced when the algorithm approaches convergence. Then Eq.(8) is given as follows

$$
\vec{f}=(\mathrm{m}-s) \times\left(\frac{\vec{\nabla} s}{|\vec{\nabla} s|^{2}+\alpha^{2}(\mathrm{~s}-m)^{2}}+\frac{\vec{\nabla} m}{|\vec{\nabla} m|^{2}+\alpha^{2}(\mathrm{~s}-m)^{2}}\right)
$$

Thirion's Demons method can be described with the flowchart (Fig. 5). The algorithm is iterative and runs for specified number of iterations. The problem with big deformations can be solved with multi-resolution approach, which drives the registration process from coarse to fine fashion. Depending on the deformation, four resolution levels are usually enough.

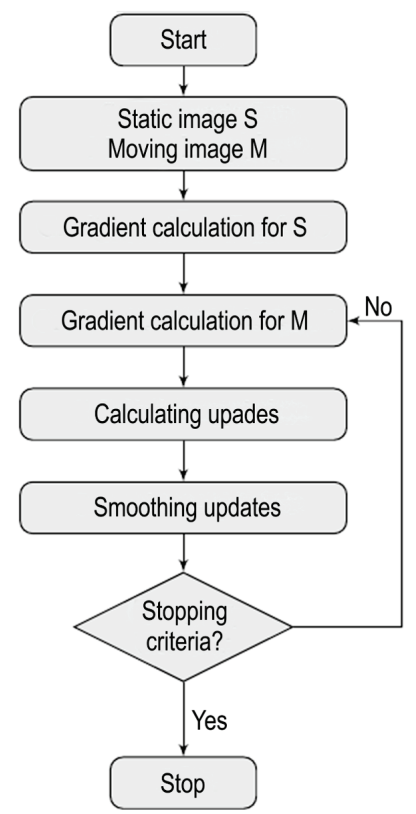

Figure 5 Flowchart of Demons registration algorithm

Thirion's Demons uses the optical flow constant intensity assumption which is satisfied only for small deformations. This can be a problem when used in settings which include large deformations, for example in the case of adaptive radiation therapy. Big displacements break the intensity constancy assumption and lead to poor registration results. Multi-resolution approach can be applied for handling this problem, and leads the optimization problem into a global optimum.

\section{RESULTS}

The common starting point in the registration evaluation is to use synthetic images. Synthetic images have ground-truth information about deformation which is not available in many practical uses. This data can give us the overview about the registration quality, especially if we can simulate deformation which is physically valid for clinical use.

In this part we will show the performance of two algorithms in a case of motion discontinuities. In a case of non-rigid image registration, the validation of results is a challenging problem. Since registration is an ill-posed problem, there should be infinitely many mappings that can be a solution to the problem. Our interest is only in cases where it is physically consistent according to type of tissue involved. For this evaluation we start with a well-known method for the image registration: FFD B- 
spline algorithm as described in Rueckert [3]. For all consequent simulations in this part, we use MATLAB environment for easy prototyping and comparisons between different algorithms.

For the quantitative analysis of the obtained deformation field was used Average Angular Error (AAE) [16]. AAE can be calculated with the following:

$$
A A E=\frac{1}{N} \sum_{i=1}^{N} \arccos \left(\frac{u_{1, i} u_{1, i}^{g t}+u_{2, i} u_{2, i}^{g t}}{\sqrt{u_{1, i}^{2}+u_{2, i}^{2}+1} \sqrt{u_{1, i}^{2, g t}+u_{2, i}^{2, g t}+1}}\right)
$$
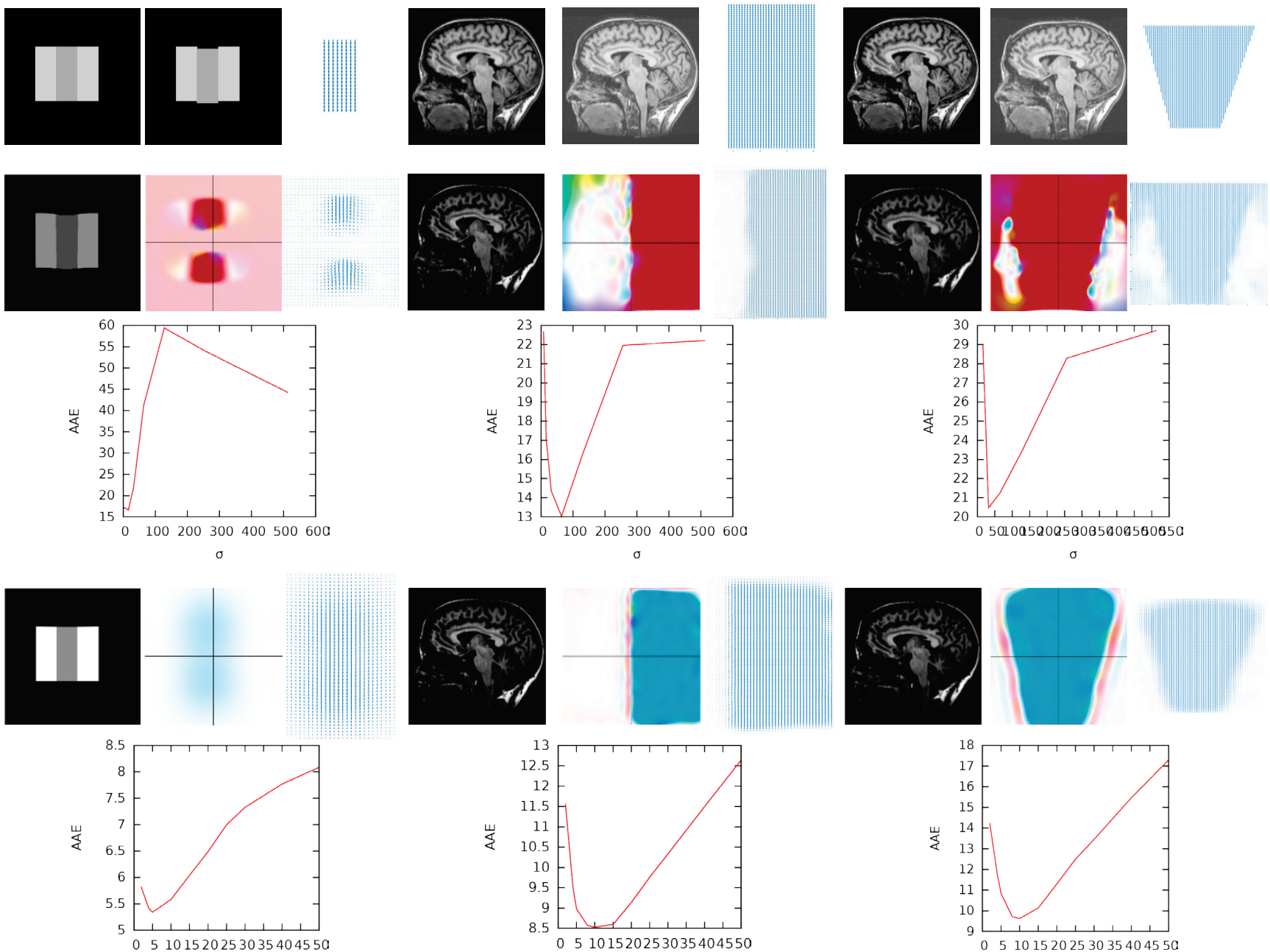

Figure 6 Evaluation results for FFD and Demons registration. First row: image to be registered and corresponding ground truth flow; second row: registration with FFD algorithm, third row: change of AAE for FFD experiments; fourth row: registrations with Demons algorithm, fifth row: change of AAE for Demons experiments

Evaluation of FFD registration will be carried for synthetic blocks and synthetic example of brain MR images. There are three synthetic examples: blocks, vertical motion and vertical shear motion. Moving image is obtained by shifting the corresponding part of fixed image 5 pixels downwards for each experiment. Then this image was registered with the original one using the FFD with different control points spacing, and Demons algorithm with different sigma values. For the evaluation we use the following control point neighbourhoods given in pixels $6 \times 6,9 \times 9,15 \times 5,26 \times 26,48 \times 48,92 \times 92$, for vertical shear motion and vertical motion, and additional for blocks $132 \times 132$ and $260 \times 260$, wherein other parameters are kept unchanged.
Fig. 6 shows the result of registration. In the first row, the pair of images to be registered is shown, and the ground truth motion field for each pair. Registration results for FFD algorithm is given in Fig. 6 in second row, deformed image and motion field, respectively. For each registration, Average Angle Error (AAE) was calculated and results are shown in Fig.6 in the third row.

For the Demons algorithm, the following filter parameter was used: $2,5,10,15,25,30,35,40$ and 45 given in pixels. For each simulation other parameters were kept unchanged. Registration results are given in the fourth row of Fig. 6, while Average Angle Error (AAE) was calculated and results are shown in the sixth row in the same figure. 
From the results for block image it can be seen that both algorithms have problems with recovering the true motion. By comparing the registration results and the motion field we can see that FFD algorithm cannot reproduce the true motion in the middle rectangle. Demons can give better visual results, but the motion around the edges is not sharp and discontinuities are not well preserved. This is visible from the AAE, i.e. Demons gives lower value than FFD. For the other two experiments, discontinuity preserving is better for both algorithms. From the visual inspection and from the AAE it can be seen that Demons for this type of images produce better registrations.

It is visible from all experiments that increasing the resolution of control points corresponds to lower AAE, but not necessarily improved registration results in the region where discontinuities lie, and also introduce some errant registrations in the right part of image on the skull edge. Also lower control point resolutions in this example cannot truly align the right part of image to its correct position, and it can be seen that, for some pixels, it is lower than true position. Better deformation field pushes some regions in their correct position, but result is unacceptable, because the deformation on the edges and also wrong results are propagated on the left side where deformation should be zero. From color rendering of the motion field onto images one can see that the difference on the left side of images and in the region where discontinuity is located, is small but noticeable.

As we can see from the results for vertical wedge-like motion, the registered image is pretty well matched except for the left and right part of the image. The result for Demons is better, only the region with wedge undergoes the motion and small motion is visible near the discontinuities.

\section{CONCLUSION}

Image registration plays an important role in today's clinical practice. Recovering transformation which maps one image into the space of the second one is generally a tedious task. The need for a clinical procedure which includes the organ motion has lead to developing the registration algorithms which take into account when one organ slides along each other. This case needs special algorithms which incorporate discontinuities into the cost function.

Free Form Deformation and Demons algorithm were evaluated with synthetically deformed images where deformation is previously known. Average Angle Error was used as the evaluation measure. Results in this work show the performance of FFD and Demons algorithm for synthetic images with discontinuities.

From the AAE measure and also from visual inspection one can see that Demons algorithm performs better for this type of motion. One can see that beside good local support, FFD algorithm gives worse results than Demons for tested pairs of MR images, as well as synthetic images. On the other side, Demons has lower computational requirements than FFD and better performance in our experiments. Therefore, it is recommended to use Demons algorithm due to its speed and simplicity and also good results in most cases.

\section{REFERENCES}

[1] Zitova, B. \& Flusser, J. (2003). Image registration methods:a survey. Image and Vision Computing, 21, 9771000. https://doi.org/10.1016/S0262-8856(03)00137-9

[2] Thirion, J. P. (1998). Image matching as a diffusion process: an analogy with maxwell's demons. Medical Image Analysis, 2(3), 243-260. https://doi.org/10.1016/S1361-8415(98)80022-4

[3] Rueckert, D., Sonoda, L. I., Hayes, C., Hill, D. L. G., Leach, M. O. \& Hawkes, D. J. (1999). Nonrigid registration using free-form deformations: application to breast MR images. IEEE Transactions on Medical Imaging, 18(8), 712-721. https://doi.org/10.1109/42.796284

[4] Muyan-Özçelik, P., Owens, J. D., Xia, J. \& Samant, S. S. (2008). Fast Deformable Registration on the GPU: A CUDA Implementation of Demons. Proceedings of the 2008 International Conference on Computational Sciences and Its Applications, IEEE Computer Society,Washington, DC, USA. https://doi.org/10.1109//CCSA.2008.22

[5] Pennec, X., Cachier, P. \& Ayache, N. (1999). Understanding the "Demon's algorithm": 3D non-rigid registration by gradient descent. Medical Image Computing and Computer-Assisted Intervention (MICCAI), 1679, 597605, September 19-22. https://doi.org/10.1007/10704282_64

[6] Gu, X., Pan, H., Liang, Y., Castillo, R., Yang, D., Choi, D., Castillo, E., Majumdar, A., Guerrero, T. \& Jiang, S. B. (2010). Implementation and evaluation of various demons deformable image registration algorithms on a GPU, Physics in Medicine and Biology. https://doi.org/10.1088/0031-9155/55/1/012

[7] Zhao, L. \& Jia, K. (2015). Deep Adaptive Log-Demons: Diffeomorphic Image Registration with Very Large Deformations. Computational and Mathematical Methods in Medicine, vol. 2015, Article ID 836202, 16 pages. https://doi.org/10.1155/2015/836202

[8] Shi, W., Xiahai, X., Pizarro, L., Bai, W., Wang, H., Tung Kai-Pin, Edwards, P. \& Rueckert, D. (2012). Registration Using Sparse Free-Form Deformations, Medical Image Computing and Computer-Assisted Intervention. MICCAI 2012: $15^{\text {th }}$ International Conference, Proceedings, Part II, Nice, France, October 1-5.

[9] Yang, G. Z., Hawkes, D. J., Rueckert, D., Noble, A. \& Taylor, C. (2009). Demons Algorithm for Image Registration with Locally Adaptive Regularization, Lecture Notes in Computer Science, Springer.

[10] Tustison, N. J. \& Avants, B. B. (2012). Diffeomorphic Directly Manipulated Free-Form Deformation Image Registration via Vector Field Flows. Proceedings Biomedical Image Registration: $5^{\text {th }}$ International Workshop, WBIR 2012, Nashville, TN, USA, July 7-8, 2012. https://doi.org/10.1007/978-3-642-31340-0_4

[11] Duchon, J. (1976). Interpolation des functions de deux variables suivant le principes de la exion des plaques mines. RAIRO Analyse Numerique, 10, 512.

[12] Meinguet, J. (1979). Multivariate interpolation at arbitrary points made simple. Zeitschrift für angewandte Mathematik und Physik, 30, 292-304. https://doi.org/10.1007/BF01601941

[13] Forsey, D. R. \& Bartels, R. H. (1988). Hierarchical b-spline refinement. Computer Graphics, 22, 205-212. https://doi.org/10.1145/378456.378512

[14] Wahba, G. (1990). Spline Models for Observational Data. CBM S - NSF Regional Conference Series in Applied Mathematics / Soc for Industrial \& Applied Math. https://doi.org/10.1137/1.9781611970128

[15] Wang, H., Dong, L., O’Daniel, J., Mohan, R., Garden, A. S., Kian Ang, K., Kuban, D. A., Bonnen, M., Chang, J. Y. \& Cheung, R. (2005). Validation of an accelerated 'demons' 
algorithm for deformable image registration in radiation therapy. Physics in Medicine and Biology, 50(12), 2887. https://doi.org/10.1088/0031-9155/50/12/011

[16] Pérez, J. S., Meinhardt-Llopis, E. \& Facciolo, G. (2013).

TV-L1 Optical Flow Estimation. Image Processing on Line, 3, 137-150. https://dx.doi.org/10.5201/ipol.2013.26

Contact information:

Emir SKEJIĆ, Associate Professor PhD

Faculty of Electrical Engineering,

University of Tuzla

Franjevačka 2

75000 Tuzla, Bosnia and Herzegovina

Phone: +38735259600

Fax: +38735259617

E-mail: emir.skejic@untz.ba

Damir DEMIROVIĆ, Assistant Professor PhD

Faculty of Electrical Engineering,

University of Tuzla

Franjevačka 2

75000 Tuzla, Bosnia and Herzegovina

Phone: +38735259600

Fax: +38735259617

E-mail: damir.demirovic@untz.ba

Dušan GLEICH, Full Professor PhD

Faculty of Electrical Engineering and Computer Science,

University of Maribor

Smetanova ulica 17

2000 Maribor, Slovenia

Phone: +386 22207128

Fax: +386 22207272

E-mail: dusan.gleich@um.si

Amira ŠERIFOVIĆ-TRBALIĆ, Assistant Professor PhD

Faculty of Electrical Engineering,

University of Tuzla

Franjevačka 2

75000 Tuzla, Bosnia and Herzegovina

Phone: +38735259600

Fax: +38735259617

E-mail: amira.serifovic@untz.ba 